\title{
Densidade da madeira de árvores em savanas do norte da Amazônia brasileira
}

\author{
Sewbert Rodrigues JATI ${ }^{1}$, Philip Martin FEARNSIDE ${ }^{2}$, Reinaldo Imbrozio BARBOSA ${ }^{3 *}$ \\ Instituto Nacional de Pesquisas da Amazônia - INPA, Curso de Pós-graduação em Biologia de Água Doce e Pesca Interior. Av. André Araújo, 2936, Petrópolis, CEP: $69067-375$. \\ Manaus, Amazonas, Brasil. sewbert@gmail.com \\ 2 Instituto Nacional de Pesquisas da Amazônia - INPA, Coordenação de Dinâmica Ambiental - CDAM. Av. André Araújo, 2936, Petrópolis, CEP: 69067-375. \\ Manaus, Amazonas, Brasil. pmfearn@inpa.gov.br \\ 3 Instituto Nacional de Pesquisas da Amazônia - INPA, Coordenação de Dinâmica Ambiental - CDAM, Núcleo de Pesquisas de Roraima - NPRR. Rua Coronel Pinto, 315, Centro, \\ CEP: 69301-150. Boa Vista, Roraima, Brasil. \\ * Autor Correspondente: reinaldo@inpa.gov.br
}

\section{RESUMO}

Densidade da madeira (DM) é uma variável importante para estimativas de estoques de carbono arbóreo em ecossistemas terrestres. Este tema é pobremente investigado em áreas de savana da Amazônia brasileira. O objetivo deste estudo foi investigar a DM das oito principais espécies arbóreas que ocorrem na savana aberta de Roraima, a maior área de savana do norte do bioma Amazônia. Foram verificadas as variaçôes na DM em função da espécie e dos diferentes diâmetros observados ao longo da dimensão vertical de 75 indivíduos amostrados em seis sítios de coleta. Foi utilizado o método direto para obtenção de peças de madeira do fuste e da copa. Os resultados indicaram discrepância significativa interespecífica, sendo Roupala montana Aubl. a espécie de maior DM média $\left(0,674 \mathrm{~g} \mathrm{~cm}^{-3}\right)$. Foi detectado que existe variaçáo significativa da DM entre as peças do fuste e da copa, independente da espécie e do sítio de coleta. A densidade da madeira de peças da copa com diâmetro entre 5 e $10 \mathrm{~cm}$ pode ser utilizada como preditora da DM média do indivíduo arbóreo. Nós concluimos que a DM das oito espécies arbóreas investigadas possui variabilidade interespecífica, com discrepâncias entre a DM do fuste e das partes lenhosas da copa. As distinçóes aqui detectadas devem ser considerados como uma importante ferramenta para melhorar as estimativas de estoque de carbono em áreas de savanas na Amazônia.

PALAVRAS-CHAVE: biomassa, carbono, cerrado, lavrado, Roraima.

\section{Wood density of trees in savannas of the northern Brazilian Amazon}

\section{ABSTRACT}

Wood density (WD) is an important variable for estimating tree carbon stocks in terrestrial ecosystems. WD has been little studied in savanna areas in the Brazilian Amazon. The aim of this study was to investigate variation in WD in eight tree species that occur in the open savannas of Roraima, the largest savanna area in the northern Brazilian Amazon. Variations were observed in WD in relation to species and diameter along the vertical dimension of 75 individuals sampled at six study sites. We used the direct method to obtain wood pieces from the stem and crown. The results indicated significant interspecific differences. Roupala montana Aubl. was the species with highest WD $\left(0.674 \mathrm{~g} \mathrm{~cm}^{-3}\right)$. Significant variation in WD was found between the stem and the woody parts of the crown, irrespective of species or sampling site. The wood density of branchs with diameter between 5 and $10 \mathrm{~cm}$ can be used as a predictor of individual WD for the whole tree. We conclude that the WD of the eight tree species investigated has interspecific variability and that there is a discrepancy between the WD of stem and the woody parts of the crown. Distinctions detected here provide an important tool for improving tree carbon estimates in Amazonian savannas.

KEYWORDS: biomass, carbon, cerrado, lavrado, Roraima. 


\section{INTRODUÇÃO}

A densidade da madeira (DM) é expressa pela razão entre a massa seca e o volume verde de uma unidade amostral (Bowyer et al. 2007). Muitas vezes é mencionada como "densidade básica", ou o peso seco dividido pelo volume da madeira saturada de água. A relação entre massa e volume é um dos índices mais simples e importantes para avaliaçáo das propriedades funcionais da madeira, refletindo também diferentes atributos da história de vida de espécies arbóreas (Muller-Landau 2004; Williamson e Wiemann 2011). A idade do indivíduo e as dimensôes radiais e verticais do caule são variáveis importantes na construção de um valor médio para a DM de uma espécie (Williamson e Wiemann 2010a; Sarmiento et al. 2011). Fatores como nichos ecológicos seletivos (Wittmann et al. 2006), estágios de sucessão (Wiemann e Williamson 1988) ou sítios latitudinais (Woodcock 2000; Wiemann e Williamson 2002; Swenson e Enquist 2007) também influenciam na formação de valores da DM no nível da espécie e da comunidade arbórea.

$\mathrm{O}$ entendimento das variaçôes da $\mathrm{DM}$ em função das espécies e ambientes vem sendo um instrumento chave para a transformação de dados de volume de madeira em biomassa (Reyes et al. 1992; Fearnside 1997; Chave et al. 2005). Esta finalidade é de grande importância, pois resulta na ampliaçáo do conhecimento da dinâmica dos estoques de carbono terrestres regionalizados, tendo aplicação direta nos acordos internacionais sobre mudanças climáticas globais (IPCC 2006). No caso da Amazônia, embora o número de estudos dedicados à avaliaçáo da DM tenha aumentado desde o final dos anos 1990, a grande maioria são de compilações ou investigaçóes que abordam diferenças regionais ou filogenéticas de espécies de árvores de sistemas florestais (Fearnside 1997; Baker et al. 2004; Nogueira et al. 2005; Chave et al. 2006; Nogueira et al. 2007). As investigaçôes em sistemas náo florestais de baixa biomassa como campinas (Barbosa e Ferreira 2004) e savanas (Barbosa e Fearnside 2004) são raras, apesar destes sistemas ocuparem ca. $200.000 \mathrm{~km}^{2}$ ou $5 \%$ de toda a área do bioma Amazônia (FUNCATE 2006) .

Do conjunto de sistemas náo florestais amazônicos, a savana de Roraima, denominada localmente como lavrado, se destaca por ocupar $42.706 \mathrm{~km}^{2}$ na região de fronteira com a Guiana e Venezuela (Barbosa e Campos 2011). Deste universo, pouco mais de $26.000 \mathrm{~km}^{2}$ (61\%) são de fitofisionomias de savana aberta com $<10 \%$ de cobertura de árvores (Barbosa et al. 2007). Várias espécies arbóreas são comuns em toda a área da savana de Roraima, com distribuição e abundância dependentes de condicionantes ambientais (Miranda et al. 2002; Barbosa et al. 2005). Estimar a DM destas espécies favorece aos ajustes nos cálculos de biomassa e carbono do componente arbóreo local, pois estabelece distinçóes regionais que representam esta grande área de savana do norte da
Amazônia. Esta tem sido uma recomendação comum do Painel Intergovernamental sobre Mudanças Climáticas (IPCC 2006) no sentido de se obter dados regionais mais realísticos com base em um maior número de espécies avaliadas através de metodologias normalizadas (Nogueira et al. 2008; Williamson e Wiemann 2010b).

Neste estudo de caso, nosso objetivo foi investigar a DM das principais espécies arbóreas da savana aberta de Roraima que ocorrem de forma comum por toda esta regiáo do extremo norte da Amazônia. Para tanto, nós verificamos variaçôes na DM em funçáo da espécie e ao longo da dimensão vertical de cada indivíduo, usando como referência classes de diâmetro das partes lenhosas do fuste e da copa. O estudo visou obter um ganho no entendimento do funcionamento ecológico e melhorar a base do cálculo de carbono arbóreo de savanas abertas da Amazônia, propiciando ajustes regionais necessários aos valores relatados na Segunda Comunicação Nacional do Brasil à Convenção-Quadro das Naçôes Unidas sobre Mudança do Clima (BRASIL-MCT 2010).

\section{MATERIAL E MÉTODOS}

\section{Sítios de coleta}

Foram selecionados seis sítios amostrais na savana de Roraima em diferentes zonas latitudinais. Todos fazem parte do mesmo tipo climático (Aw pela classificação de Köppen), embora sejam distintos por características ambientais gerais, com chuva anual descrescente de sudoeste para nordeste (1700 $\mathrm{mm}=>1100 \mathrm{~mm})$ e altitude crescente no mesmo sentido (Barbosa 1997; Barbosa et al. 2007):

(1) Mucajaí (02॰ 31’ 46” N; 60 52' 57” W; 75 m acima do nível do mar) e Boa Vista (02。 46' 55" N; 60 41' 38" W; $80 \mathrm{~m}$ ): representam as áreas de savana de baixa altitude situadas ao sul da Formaçáo Boa Vista (BRASIL-MME 1975). $\mathrm{Na}$ grande maioria dos casos são savanas graminosas do tipo campo limpo e sujo, em mosaico com savana parque (IBGE 2012). Ocupam relevos planos ou pouco ondulados.

(2) Truaru (03 20' 07” N; 60 48' 25” W; $105 \mathrm{~m}$ ) e Três Coraçôes (03 36' 03” N; 60 57’ 18” W; 145 m): áreas dispostas no centro-norte da Formação Boa Vista. Região caracterizada por relevo com maior grau de ondulação onde a savana graminosa predomina nas partes mais baixas, e as savanas parque ou de baixa arborização são destacadas nos relevos mais acentuados.

(3) Uiramutã (040 36’ 01” N; 60 09’ 28” W; $535 \mathrm{~m}$ ) e Pacaraima (04 28’ 52’ N; 61 09' 22” W; 915 m): sítios caracterizados por savanas de alta altitude concentradas ao longo de toda a Formação Roraima (Pré-Cambriano). Correspondem às savanas estépicas graminosas e parquedas situadas em relevo ondulado de alta altitude (IBGE 2012). 


\section{Seleção de espécies e indivíduos}

As espécies arbóreas selecionadas para este estudo são as mais comuns e de maior abundância em toda a savana aberta de Roraima: Byrsonima crassifolia (L.) Kunth. (Malpighiaceae; Registro de Conferência no Herbário INPA 213927), $B$. coccolobifolia Kunth. (INPA 213929), Curatella americana L.f. (Dilleniaceae; INPA 175998), Antonia ovata Pohl. (Loganiaceae; INPA 201941), Bowdichia virgilioides Kunth. (Fabaceae; INPA 187149), Himatanthus articulatus (Vahl.) Woods. (Apocynaceae; UFRR 1968), Roupala montana Aubl. (Proteaceae; INPA 213171) e Xylopia aromatica (Lam.) Mart. (Annonaceae; INPA 201942). As três primeiras representam 60-70\% do número de indivíduos e $c a$. $80 \%$ do total de biomassa arbórea acima do solo nas áreas de savana aberta de Roraima (Barbosa e Fearnside 2005). Associadas com as demais espécies, formam a base do componente arbóreo desta ecorregiāo (Miranda et al. 2002; Barbosa et al. 2012a).

A seleção dos indivíduos foi realizada entre novembro/2007 e junho/2008. Para manter a independência na escolha dos indivíduos foram estabelecidos transectos em cada um dos seis sítios amostrais, aproveitando as trilhas de caminhamento previamente existentes em cada área: 2 a 3 por sítio com extensão de 300-500 m cada um. Em cada transecto foram selecionados apenas indivíduos alocados exclusivamente em uma faixa de distância de $5 \mathrm{~m}$ de cada lado da linha central de caminhamento. O critério biométrico adotado para escolha dos indivíduos amostrados foi o do diâmetro basal do caule $\left(D_{b}\right)>10 \mathrm{~cm}$, medido à $2 \mathrm{~cm}$ de altura do solo ou logo acima deste limite em funçáo de eventuais protuberâncias do caule. Esta medida foi tomada levando em consideração a transformaçáo da circunferência da base obtida por meio de uma fita métrica calibrada em centímetros. O critério biométrico basal foi adotado para que cada indivíduo amostrado fosse configurado plenamente na fase adulta, e que fosse possível obter todas as diferentes categorias de diâmetro ao longo de sua dimensão vertical. Nós não cobrimos a falta de espécies fora dos transectos em qualquer dos sítios amostrais com o objetivo de manter a independência amostral, mesmo entendendo perdas na representatividade.

\section{Desenho experimental}

A coleta das amostras foi realizada através do método direto (destrutivo), aproveitando a autorização de coleta de material botânico n ${ }^{\circ}$ 11035-1 (IBAMA/SISBIO). Cada indivíduo selecionado foi seccionado em peças de madeira no formato de discos amostrais (cerne + alburno + casca) ao longo de toda sua dimensão vertical: (1) peças do fuste - caule principal (diâmetro $(\mathrm{d})>10 \mathrm{~cm})$ e (2) peças da copa - gravetos $(1<\mathrm{d}$ $\leq 5 \mathrm{~cm})$ e galhos $(5<\mathrm{d} \leq 10 \mathrm{~cm})$. As peças da copa possuiam comprimento padráo de 6-7 cm e, as do fuste, $3-4 \mathrm{~cm}$. Esta operação foi realizada com arco de serra manual.
Da copa de cada indivíduo foram coletadas sistematicamente quatro peças de gravetos e quatro de galhos em cada vértice cardinal (Norte, Sul, Leste, Oeste). Do fuste foram extraídas peças na seguinte sequência: (1) secção basal $\left(D_{b}\right)$ de cada indivíduo, extraída à $2 \mathrm{~cm}$ de altura do nível do solo, (2) secção à $30 \mathrm{~cm}\left(\mathrm{D}_{30}\right)$ de altura do nível do solo e (3) outras secçóes do fuste $\left(\mathrm{D}_{\text {osf }}\right)$, obtidas em partes proporcionalmente distanciadas até alcançar o início dos galhos. A altura das secçôes foi padronizada em função do tamanho das árvores da savana local que, geralmente, não ultrapassam a altura de 6-8 m (Barbosa e Fearnside 2005). Foram utilizadas secçôes circulares com casca ao longo de todas as classes de diâmetro para que a variaçấo radial e vertical entre indivíduos e espécies pudesse ser totalmente representada (Nogueira et al. 2005).

A DM foi calculada de forma a expressar a massa (peso seco) de cada peça por unidade de volume verde com conteúdo de umidade estimado como estando acima do ponto de saturação das fibras no ato do corte de cada peça em campo (Bowyer et al. 2007). Desta forma, cada peça de madeira, ou fraçáo correspondente a uma cunha, foi imediatamente imersa em uma proveta graduada para aferiçáo do deslocamento de água relacionado ao volume verde da peça $\left(\mathrm{cm}^{3}\right)$. Após este procedimento, todas as peças seguiram ao laboratório para secagem em estufa à $100 \pm 5^{\circ} \mathrm{C}$ até peso constante $(\mathrm{g}-0 \%$ umidade). A fração casca foi pesada separadamente da fração cerne + alburno para o cálculo da porcentagem entre estas duas fraçóes por classe de diâmetro e espécie com base no peso seco $(\mathrm{g})$.

\section{Análise dos dados}

Teste de Lillefors foi usado para verificar a normalidade da distribuição dos dados. Foi utilizada ANOVA (fator único; $\alpha=0,05)$ para avaliar a variaçāo da $\mathrm{DM}$ ao longo da dimensão vertical das diferentes categorias de diâmetro entre as peças do fuste $\left(\mathrm{D}_{\mathrm{b}} ; \mathrm{D}_{30} ; \mathrm{D}_{\text {osf }}\right)$ e da copa (galhos e gravetos) tomando como base os resultados individuais de cada amostra. Considerou-se a média aritimética das repetiçóes como resultado individualizado de cada fraçáo. Para galhos e gravetos, este procedimento foi independente da posiçáo cardinal. Múltiplas comparaçôes entre as diferentes frações foram realizadas pelo Teste de Tukey $(\alpha=0,05)$, independente da espécie e do sítio amostral.

Para verificar diferenças entre as espécies, nós calculamos o valor médio ponderado da DM e da porcentagem de casca de cada indivíduo amostrado tomando como base a proporçáo média de biomassa de cada classe de diâmetro para cada uma das espécies investigadas na savana de Roraima (Material Suplementar de Barbosa et al. 2012b). Neste caso, as classes consideradas foram fuste (média simples de todas as secçôes do caule), galhos e gravetos. Desta forma, foi obtido um único valor ponderado de DM e de porcentagem de casca para cada indivíduo. $\mathrm{O}$ conjunto dos dados de DM foi separado por 
espécie e aplicada ANOVA (fator único) seguida do Teste de Tukey $(\alpha=0,05)$.

Foi aplicada a correlaçấo de Pearson (r) entre os resultados de DM ponderada de cada indivíduo e suas respectivas classes do fuste e da copa. Uma regressão linear simples foi gerada com o objetivo de verificar o uso de peças de menor diâmetro como preditoras da DM média ponderada de cada indivíduo (variável dependente).

\section{RESULTADOS}

A amostragem dos 75 indivíduos com $\mathrm{D}_{\mathrm{b}}>10 \mathrm{~cm}$ das oito espécies de árvores mais abundantes da savanna aberta de Roraima indicou que a DM náo variou entre as classes de diâmetro do fuste $\left(\mathrm{D}_{\mathrm{b}}, \mathrm{D}_{30}\right.$ e $\left.\mathrm{D}_{\text {osf }}\right)$, mas todas estas foram significativamente maiores em relaçáo as da copa (galhos e gravetos) (ANOVA; $\mathrm{F}_{4,339}=31,55 ; \mathrm{P}<0,00001$ ) (Figura 1).

A DM entre as espécies diferiu significativamente (ANOVA; $\mathrm{F}_{7,67}=12,74 ; \mathrm{P}<0,00001$ ), com o maior valor sendo observado para $R$. montana $\left(0,674 \mathrm{~g} \mathrm{~cm}^{-3}\right)$ e o menor para $C$. americana $\left(0,525 \mathrm{~g} \mathrm{~cm}^{-3}\right)$. A média geral de todas as espécies, tomando como base as classes de diâmetro do fuste e da copa, foi de $0,592 \mathrm{~g} \mathrm{~cm}^{-3}\left(0,427\right.$ a $\left.0,716 \mathrm{~g} \mathrm{~cm}^{-3}\right)$.

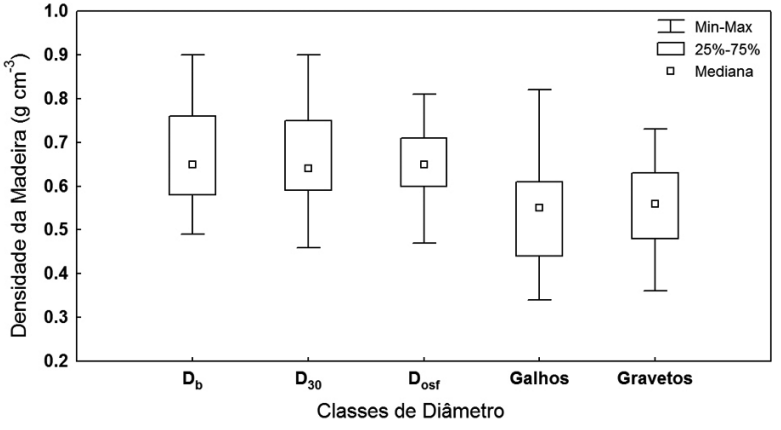

Figura 1 - Média ponderada da densidade da madeira $\left(\mathrm{g} \mathrm{cm}^{-3}\right)$ para todas as classes de diâmetro do fuste $\left(D_{b}, D_{30}\right.$ e $\left.D_{\text {osf }}\right)$ e da copa (galhos e gravetos) das principais espécies de árvores da savana aberta de Roraima. Valores apresentam a mediana, o primeiro e terceiro quartis, e o intervalo dos dados para 75 árvores com $D_{b}>10 \mathrm{~cm}$. Onde: $D_{b}=$ diâmetro do fuste na secção basal, $\mathrm{D}_{30}=$ diâmetro do fuste na secção à $30 \mathrm{~cm}$ do solo, $\mathrm{D}_{\text {osf }}=$ diâmetro médio das secções remanescentes do fuste, Galhos = peças da copa com diâmetro entre 5 e $10 \mathrm{~cm}$ e Gravetos = peças da copa com diâmetro entre 1 e $5 \mathrm{~cm}$.

Tabela 1 - Densidade da madeira $\left(\mathrm{g} \mathrm{cm}^{-3}\right)$ das principais espécies arbóreas da savana aberta de Roraima distintas por classes de diâmetro ao longo da dimensão vertical. Onde: Fuste $=$ peças do caule principal com diâmetro (d) $>10 \mathrm{~cm}$, Galhos = peças da copa com $5<\mathrm{d} \leq 10 \mathrm{~cm}$ e Gravetos $=$ peças da copa com $1<d \leq 5 \mathrm{~cm}$. Médias das espécies foram calculadas como uma ponderação porcentual da biomassa seca entre as classes de diâmetro, conforme descrito no texto. Letras minúsculas (linha) e maiúsculas (coluna) representam discrepâncias estatísticas, respectivamente, entre as classes de diâmetro e as espécies, no nível de 5\% (ANOVA; Teste de Tukey).

\begin{tabular}{|c|c|c|c|c|c|c|}
\hline \multirow{2}{*}{ Família } & \multirow{2}{*}{ Espécie } & \multirow{2}{*}{$\begin{array}{c}\text { Número } \\
\text { Indivíduos (1) }\end{array}$} & \multicolumn{4}{|c|}{ Densidade da Madeira $\left(\mathrm{g} \mathrm{cm}^{-3}\right)$ (2) } \\
\hline & & & Fuste & Galhos & Gravetos & Média da Espécie \\
\hline Annonaceae & $X$. aromatica & $6(67)$ & $\begin{array}{c}0,649 \mathrm{~b} \\
(0,498-0,872)\end{array}$ & $\begin{array}{c}0,564 \mathrm{a} \\
(0,439-0,629)\end{array}$ & $\begin{array}{c}0,578 \mathrm{ab} \\
(0,498-0,644)\end{array}$ & $\begin{array}{c}0,605 \mathrm{BC} \\
(0,541-0,644)\end{array}$ \\
\hline Apocynaceae & H. articulatus & $7(83)$ & $\begin{array}{c}0,636 \mathrm{~b} \\
(0,492-0,813)\end{array}$ & $\begin{array}{c}0,464 \mathrm{a} \\
(0,290-0,655)\end{array}$ & $\begin{array}{c}0,440 \mathrm{a} \\
(0,356-0,577)\end{array}$ & $\begin{array}{c}0,544 \mathrm{AB} \\
(0,497-0,607)\end{array}$ \\
\hline Dilleniaceae & C. americana & $18(213)$ & $\begin{array}{c}0,674 \mathrm{~b} \\
(0,354-0,904)\end{array}$ & $\begin{array}{c}0,442 \mathrm{a} \\
(0,314-0,569)\end{array}$ & $\begin{array}{c}0,466 \mathrm{a} \\
(0,357-0,587)\end{array}$ & $\begin{array}{c}0,525 \mathrm{~A} \\
(0,427-0,600)\end{array}$ \\
\hline Fabaceae & B. virgilioides & $8(119)$ & $\begin{array}{c}0,689 \mathrm{~b} \\
(0,485-0,900)\end{array}$ & $\begin{array}{c}0,462 \mathrm{a} \\
(0,281-0,795)\end{array}$ & $\begin{array}{c}0,568 \mathrm{a} \\
(0,436-0,704)\end{array}$ & $\begin{array}{c}0,580 \mathrm{ABC} \\
(0,520-0,646)\end{array}$ \\
\hline Loganiaceae & A. ovata & $7(71)$ & $\begin{array}{c}0,625 \mathrm{~b} \\
(0,573-0,790)\end{array}$ & $\begin{array}{c}0,552 \mathrm{a} \\
(0,504-0,665)\end{array}$ & $\begin{array}{c}0,594 \mathrm{ab} \\
(0,523-0,646)\end{array}$ & $\begin{array}{c}0,596 \mathrm{BC} \\
(0,551-0,662)\end{array}$ \\
\hline \multirow[t]{2}{*}{ Malpighiaceae } & B. coccolobifolia & $9(111)$ & $\begin{array}{c}0,654 \mathrm{~b} \\
(0,470-0,786)\end{array}$ & $\begin{array}{c}0,587 \mathrm{a} \\
(0,494-0,672)\end{array}$ & $\begin{array}{c}0,613 \mathrm{ab} \\
(0,550-0,679)\end{array}$ & $\begin{array}{c}0,615 \mathrm{CD} \\
(0,589-0,647)\end{array}$ \\
\hline & B. crassifolia & $13(148)$ & $\begin{array}{c}0,669 \mathrm{~b} \\
(0,502-0,896)\end{array}$ & $\begin{array}{c}0,611 \mathrm{ab} \\
(0,523-0,718)\end{array}$ & $\begin{array}{c}0,594 \mathrm{a} \\
(0,493-0,732)\end{array}$ & $\begin{array}{c}0,619 \mathrm{CD} \\
(0,543-0,714)\end{array}$ \\
\hline Proteaceae & R. montana & $7(78)$ & $\begin{array}{c}0,681 \mathrm{a} \\
(0,544-0,878)\end{array}$ & $\begin{array}{c}0,665 \mathrm{a} \\
(0,610-0,821)\end{array}$ & $\begin{array}{c}0,672 \mathrm{a} \\
(0,565-0,726)\end{array}$ & $\begin{array}{c}0,674 \mathrm{D} \\
(0,641-0,716)\end{array}$ \\
\hline \multicolumn{2}{|c|}{ Média das Classes } & 75 (890) & $\begin{array}{c}0,660 \mathrm{~b} \\
(0,354-0,904)\end{array}$ & $\begin{array}{c}0,543 a \\
(0,281-0,821)\end{array}$ & $\begin{array}{c}0,566 \mathrm{a} \\
(0,356-0,732)\end{array}$ & $\begin{array}{c}0,592 \\
(0,427-0,716)\end{array}$ \\
\hline
\end{tabular}

(1) Entre parênteses o total de peças amostradas por espécie; (2) Entre parênteses a variação dos valores de densidade da madeira observados em cada classe de diâmetro ao longo da dimensão vertical. 
A concentração de casca foi decrescente entre as peças do fuste e da copa, exceto para X. aromatica (Tabela 2). Considerando a média ponderada entre as diferentes partes do fuste e da copa, a maior concentração de casca foi observada em $R$. montana $(35,8 \%)$ e a menor em $A$. ovata $(26,4 \%)$.

A média ponderada da DM de cada indivíduo foi significativamente correlacionada com DM das peças derivadas dos galhos da copa com diâmetro entre 5 e $10 \mathrm{~cm}(\mathrm{r}$ $=0,83 ; \mathrm{P}<0,00001)$. O coeficiente de determinação $\left(\mathrm{R}^{2}\right) \mathrm{da}$ regressão linear entre estas duas variáveis foi de 0,69 (Figura 2).

Tabela 2 - Porcentagem de casca (\%), por classes de diâmetro, determinado para as principais espécies arbóreas da savana aberta de Roraima. Onde: Fuste $=$ peças do caule principal com diâmetro $(\mathrm{d})>10 \mathrm{~cm}$, Galhos $=$ peças da copa com $5<d \leq 10 \mathrm{~cm}$ e Gravetos = peças da copa com $1<d \leq 5 \mathrm{~cm}$. Médias das espécies foram calculadas como uma ponderação porcentual da biomassa seca entre as classes de diâmetro, da mesma forma como descrito no texto para densidade da madeira.

\begin{tabular}{llcccc}
\hline \multirow{2}{*}{ Família } & Espécie & \multicolumn{4}{c}{ Porcentagem de Casca (\%) } \\
\cline { 3 - 6 } & & Fuste & Galhos & Gravetos & $\begin{array}{c}\text { Média } \\
\text { Ponderada }\end{array}$ \\
\hline Annonaceae & X. aromatica & 31,0 & 32,8 & 27,3 & 30,4 \\
Apocynaceae & H. articulatus & 30,0 & 25,9 & 22,9 & 27,6 \\
\hline Dilleniaceae & C. americana & 34,4 & 30,6 & 23,9 & 30,0 \\
Fabaceae & B. virgilioides & 35,7 & 30,5 & 26,0 & 29,7 \\
Loganiaceae & A. ovata & 28,4 & 27,9 & 20,9 & 26,4 \\
Malpighiaceae & B. coccolobifolia & 38,1 & 31,5 & 28,1 & 32,4 \\
\hline Proteaceae & R. montana & 44,1 & 35,0 & 22,6 & 35,8 \\
\hline Média & & 35,2 & 30,7 & 24,6 & 28,5 \\
\hline
\end{tabular}

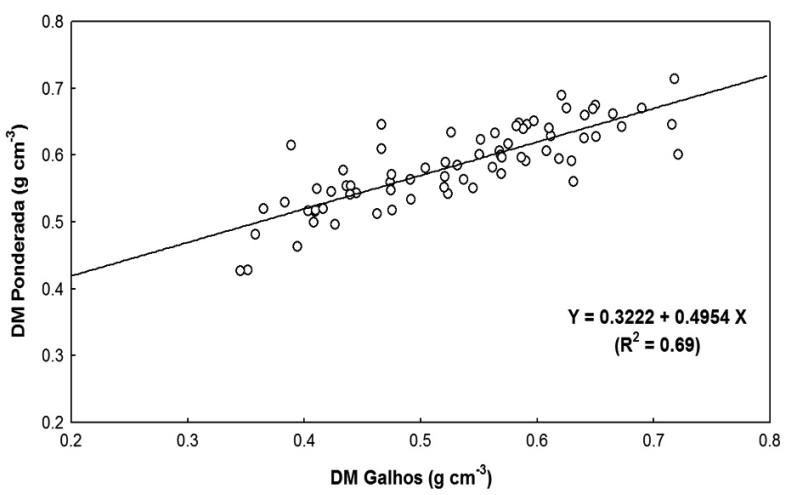

Figura 2 - Regressão linear simples indicando a relação entre a média ponderada da DM (densidade da madeira) de todas as partes lenhosas de cada indivíduo $(Y)$ e a DM de galhos $(X)$ com diâmetro entre 5 e $10 \mathrm{~cm}$ das principais espécies arbóreas da savana aberta de Roraima $(n=75)$.

\section{DISCUSSÃO}

\section{Variabilidade interespecífica}

De forma geral, os valores médios determinados para cada espécie em nosso estudo $\left(0,427-0,716 \mathrm{~g} \mathrm{~cm}^{-3}\right)$ estáo dentro do intervalo esperado para espécies e famílias tropicais (Baker $e t$ al. 2004; Chave et al. 2006), sendo similares aos observados em alguns táxons arbóreos do cerrado do Brasil Central $\left(0,35-0,75 \mathrm{~g} \mathrm{~cm}^{-3}\right)$ por Bucci et al. (2004). Da mesma forma, nosso valor médio geral $\left(0,592 \mathrm{~g} \mathrm{~cm}^{-3}\right)$ se encontra na mesma faixa de densidade da madeira determinada por Oliveira et al. (2012) para os cerradóes e cerrados típicos de Minas Gerais $\left(0,585-0,612 \mathrm{~g} \mathrm{~cm}^{-3}\right)$. Contudo, a média geral de nosso estudo é $46 \%$ maior do que o calculado com base no volume rehidratado $\left(0,404 \mathrm{~g} \mathrm{~cm}^{-3}\right)$ para a mesma regiáo por Barbosa e Fearnside (2004). Essa diferença é devido ao fato dos dois estudos terem usados medidas diferentes de densidade, com o de Barbosa e Fearnside (2004) fazendo uso da "densidade básica” por rehidratação das peças amostrais. A DM medida no atual trabalho (volume da madeira determinado com seu conteúdo de água no momento da coleta), tem vantagens e desvantagens em comparaçáo com a medida mais amplamente relatada de "densidade básica", onde as amostras de madeira são rehidratadas até a saturação antes da determinaçáo do volume. A medida utilizada aqui oferece uma representação melhor da madeira em árvores vivas, que é o valor necessário para converter as estimativas de volume realizadas em campo com base no peso seco da biomassa para fins de cálculo de estoques de carbono. Por outro lado, o conteúdo de água no momento da colheita pode estar sujeito a maior variaçáo devido a diferenças no teor de água ao longo de ciclos sazonais ou em resposta à falta ou ao excesso de água no curto prazo. Procedimentos de medição podem introduzir erros em ambos os casos: (1) no caso de densidade calculada com base no teor de água no momento da coleta, qualquer atraso entre o corte da árvore e a mediçáo do volume implica na perda de água da amostra, enquanto (2) para densidade calculada com base em amostras de madeira saturada (rehidratada), qualquer insuficiência no tempo em que as amostras são deixadas de molho resultará em amostras saturadas de forma incompleta.

Analisando os valores específicos, apenas $R$. montana pode ser definida como 'média-pesada' $\left(0,65\right.$ a $\left.0,80 \mathrm{~g} \mathrm{~cm}^{-3}\right)$ pelo sistema de classificaçáo de densidade básica da madeira utilizado por Sternadt (2001) para espécies amazônicas; as demais foram consideradas como 'média-leve' ( 0,50 a 0,65 $\left.\mathrm{g} \mathrm{cm}^{-3}\right)$. Esta constatação está diretamente relacionada à alta concentração de casca das espécies avaliadas (21-44\%). Grande proporção desta fração é comum em espécies de savana (Nygård e Elfving 2000; Barbosa e Fearnside 2004) e está relacionada a uma estratégia de proteção contra fogo e herbívoros (Larjavaara e Muller-Landau 2010; Lawes et al. 2011). Desta forma, maior ou menor proporção desta fraçáo 
pode influenciar diretamente nos valores médios no nível da espécie. Por exemplo, levando em consideraçáo que a média de DM da fração casca é $80 \%$ da DM da fração cerne + alburno em espécies florestais (Fearnside 1997), nós estimamos que medidas de DM das espécies avaliadas neste estudo seriam incrementadas em pelo menos $5-8 \%$ sem a presença da casca: peças de maior diâmetro tendo menor incremento devido à menor concentração desta fração (Tabela 2). Esta condição não seria a ideal para fins de cálculo de estoque de carbono contido na biomassa arbórea, visto que a fração casca (ca. 30\%) é muito importante nas estimativas deste componente em ecossistemas de savana. Assim sendo, embora seja recomendado que medidas de DM sejam realizadas em amostras que não contenham medula e casca devido a variaçôes nâo controladas entre indivíduos e espécies (Williamson e Wiemann 2010b), nós indicamos o uso de amostras completas (cerne + alburno + casca) para efeito de cálculos de biomassa arbórea em áreas de savana. As estimativas dos estoques de carbono arbóreo seriam mais realistas no nível da espécie e da comunidade.

\section{Variabilidade diamétrica (dimensão vertical)}

Nossos resultados implicam que peças de menor diâmetro da copa possuam menor DM em relação às peças de maior diâmetro do fuste. Esse padrão é o mesmo observado em outros ecossistemas abertos da Amazônia (Barbosa e Ferreira 2004), embora resultados discordantes possam ser relatados para DM de espécies florestais com DAP $>10 \mathrm{~cm}$ (Chave et al. 2004). De qualquer forma, nossos valores médios de densidade da madeira, tomando como base a proporção de biomassa entre as diferentes classes de diâmetro, indicam ser mais realistas para estimativas de estoques de carbono arbóreo. Isto porque, em geral, o valor de DM adotado nos cálculos de biomassa são derivados de estudos que utilizaram amostras de um único ponto do fuste (e.g. altura do peito) (Sarmiento et al. 2011). Contudo, o uso exclusivo da média entre várias medidas de DM ao longo do fuste pode inferir em uma redução de 5,3\% no valor da DM comumente utilizada para espécies florestais da Amazônia (Nogueira et al. 2005). Em nosso caso, levando em conta que árvores de savana possuem fustes menores e maior proporção de biomassa de galhos e gravetos (e.g. $-60 \%$ para espécies da savana de Roraima), os valores podem conter distorçôes ainda maiores. Por exemplo, usando apenas a média aritimética entre as classes de diâmetro do fuste $\left(D_{b}, D_{30}\right.$ e $\left.D_{\text {osf }}\right)$ avaliadas neste estudo, verificamos que o uso individual de qualquer uma delas estabeleceria um valor $15-17 \%$ maior em relação ao valor de DM ponderado para todas as partes lenhosas. Isso provocaria superestimativas quando da transformaçáo de dados de volume em biomassa para toda esta regiāo.

Para evitar o uso convencional de valores exclusivos do fuste e reduzir os danos no nível do indivíduo devido à adoção do método destrutivo total, nós sugerimos a aplicação de equaçôes alométricas que possibilitem a coleta de peças menores preditoras da DM ponderada de todas as partes do indivíduo. Embora o uso de peças com diâmetros menores venha sendo desaconselhado por questóes relacionadas ao desenvolvimento ontogenético dos ramos que podem provocar valores não representativos (Williamson e Wiemann 2010b), este procedimento tem sido adotado com sucesso na averiguação de diferentes relaçôes ecológicas na Amazônia (Patiño et al. 2009). Nossas avaliaçóes indicaram que a DM de galhos com diâmetro entre 5 e $10 \mathrm{~cm}$ foi significativamente correlacionada com a DM ponderada de indivíduos arbóreos com $\mathrm{D}_{\mathrm{b}}>10 \mathrm{~cm}$, com $69 \%$ da variável dependente sendo explicada pelo modelo da Figura 2. Correlação entre medidas de densidade da madeira do fuste e dos galhos também foi determinada em árvores de florestas tropicais de Porto Rico, indicando que este tipo de relação é comum em outros sistemas tropicais (Swenson e Enquist 2008). No caso de árvores da savana de Roraima, peças da copa com $5<\mathrm{d} \leq$ $10 \mathrm{~cm}$ representam a extensão primária do fuste, indicando não apresentar os críticos problemas ontogenéticos acima relatados.

Mesmo entendendo que o modelo preditor não seja totalmente ajustado devido à dependência de outras variáveis não observadas neste estudo, a regra de coletar peças dos galhos para estimar a DM do indivíduo arbóreo através de regressão é vantajosa, independente do método de coleta (peças seccionadas ou trado de incremento). Além de evitar o uso do método destrutivo em todo o indivíduo, tenderia a reduzir erros de superestimativas nos estoques de biomassa/ carbono arbóreo devido ao uso tradicional de amostras derivadas do fuste.

\section{CONCLUSÕES}

Existe variação interespecífica da DM entre as principais espécies arbóreas da savana de Roraima. Na maioria dos casos, a DM do fuste e das partes lenhosas que formam a copa também apresentam variabilidade. Contudo, peças da copa com diâmetro entre 5 e $10 \mathrm{~cm}$ podem ser utilizadas como preditoras da DM ponderada de todas as partes lenhosas de indivíduos com $\mathrm{D}_{\mathrm{b}}>10 \mathrm{~cm}$. Os valores específicos aqui determinados devem ser considerados como uma importante ferramenta para melhorar as estimativas de estoque de carbono arbóreo em áreas de savanas amazônicas.

\section{AGRADECIMENTOS}

O projeto institucional "Ecologia e manejo dos recursos naturais da savana de Roraima" (PPI/INPA PRJ 012.18) e o Instituto Nacional de Ciência e Tecnologia dos Serviços Ambientais da Amazônia (INCT-ServAmb/MCT) apoiaram o estudo. O Exército Brasileiro permitiu o uso de seus campos militares estabelecidos próximos às cidades de Uiramutã e 
Pacaraima para coleta de material amostral. O Ministério das Comunicaçôes permitiu o uso de sua área institucional situada na periferia da cidade de Boa Vista. Claymir de Oliveira Cavalcante (UFRR/PRONAT) colaborou com as coletas de campo. O Conselho Nacional de Desenvlvimento Científico e Tecnológico (CNPq) concedeu bolsa produtividade a R. I. Barbosa e P. M. Fearnside.

\section{BIBLIOGRAFIA CITADA}

Baker, T.R.; Phillips, O.; Malhi, Y.; Almeida, S.; Arroyo, L.; Fiore, A.D.; et al. 2004. Variation in wood density determines spatial patterns in Amazonian forest biomass. Global Change Biology, 10: $545-562$.

Barbosa, R.I. 1997. Distribuição das chuvas em Roraima. In: Barbosa, R.I.; Ferreira, E.F.G.; Castellon, E.G. (Eds.). Homem, Ambiente e Ecologia no Estado de Roraima. Instituto Nacional de Pesquisas da Amazônia e Governo do Estado de Roraima, Manaus, Amazonas, p.325-335.

Barbosa, R.I.; Campos, C. 2011. Detection and geographical distribution of clearing areas in the savannas ('lavrado') of Roraima using Google Earth web tool. Journal of Geography and Regional Planning, 4: 122-136.

Barbosa, R.I.; Campos, C.; Pinto, F.; Fearnside, P.M. 2007. The "Lavrados" of Roraima: biodiversity and conservation of Brazil's Amazonian savannas. Functional Ecosystems and Communities, 1: $29-41$.

Barbosa, R.I.; Fearnside, P.M. 2004. Wood density of trees in open savannas of the Brazilian Amazon. Forest Ecology and Management, 199: 115-123.

Barbosa, R.I.; Fearnside, P.M. 2005. Above-ground biomass and the fate of carbon after burning in the savannas of Roraima, Brazilian Amazonia. Forest Ecology and Management, 216: 295-316.

Barbosa, R.I.; Ferreira, C.A.C. 2004. Densidade básica da madeira de um ecossistema de campina em Roraima, Amazônia brasileira. Acta Amazonica, 34: 587-591.

Barbosa, R.I.; Mourão Jr., M.; Casadio, G.M.L.; da Silva, S.J.R. 2012a. Reproductive phenology of the main tree species in the Roraima savanna, Brazilian Amazon. Ecotropica, 18: 81-91.

Barbosa, R.I.; Nascimento, S.P.; Amorim, P.A.F.; Silva, R.F. 2005. Notas sobre a composição arbóreo-arbustiva de uma fisionomia das savanas de Roraima, Amazônia Brasileira. Acta Botanica Brasilica, 19: 323-329.

Barbosa, R.I.; Santos, J.R.S.; Cunha, M.S.; Pimentel, T.P.; Fearnside, P.M. 2012b. Root biomass, root: shoot ratio and belowground carbon stocks in the open savannahs of Roraima, Brazilian Amazonia. Australian Journal of Botany, 60: 405-416.

Bowyer, J.L.; Shmulsky, R.; Haygreen, J.G. 2007. Forest products and wood science: an introduction. 5th ed. Blackwell Publishing, Iowa, 2007, 557p.

BRASIL-MCT. 2010. Segunda Comunicaçâo Nacional do Brasil à Convenção-Quadro das Naçóes Unidas sobre Mudança do Clima. Vol. 1. Coordenação-Geral de Mudanças Globais do Clima, Ministério da Ciência e Tecnologia, Brasília-DF, 2010, 280p.
BRASIL-MME. 1975. Projeto RADAMBRASIL - Levantamento de Recursos Naturais. Vol. 8. Ministério das Minas e Energia, Departamento Nacional de Produçáo Mineral, Rio de Janeiro, 1975, 475p.

Bucci, S.J.; Goldstein, G.; Meinzer, F.C.; Scholz, F.G.; Franco, A.C.; Bustamante, M. 2004. Functional convergence in hydraulic architecture and water relations of tropical savanna trees: from leaf to whole plant. Tree Physiology, 24: 891-899.

Chave, J.; Andalo, C.; Brown, S.; Cairns, M.A.; Chambers, J.Q.; Eamus, D.; et al. 2005. Tree allometry and improved estimation of carbon stocks and balance in tropical forests. Oecologia, 145: 87-99.

Chave, J.; Condit, R.; Aguilar, S.; Hernandez, A.; Lao, S.; Perez, R. 2004. Error propagation and scaling for tropical forest biomass estimates. Philosophical Transactions of the Royal Society B: Biological Sciences, 359: 409-420.

Chave, J.; Muller-Landau, H.C.; Baker, T.R.; Easdale, T.A.; Steege, H.T.; Webb, C.O. 2006. Regional and phylogenetic variation of wood density across 2456 Neotropical tree species. Ecological Applications, 16: 2356-2367.

Fearnside, P.M. 1997. Wood density for estimating forest biomass in Brazilian Amazonia. Forest Ecology and Management, 90: 59-87.

FUNCATE. 2006. Uso e cobertura da terra na Floresta Amazônica: Subprojeto 106/2004 PROBIO (Projeto de Conservação e Utilização Sustentável da Diversidade Biológica Brasileira). Secretaria de Biodiversidade e Florestas do Ministério de Meio Ambiente, Conselho Nacional de Desenvolvimento Científico e Tecnológico, Fundo para o Meio Ambiente Global e Banco Mundial, Brasília, DF, 2006, 93p.

IBGE. 2012. Manual técnico da vegetação brasileira: sistema fitogeográfico, inventário das formaçōes florestais e campestres, técnicas e manejo de coleçôes botânicas, procedimentos para mapeamentos. 2a ed. IBGE-Instituto Brasileiro de Geografia e Estatística, Rio de Janeiro, 2012, 275p.

IPCC. 2006. 2006 IPCC Guidelines for National Greenhouse Gas Inventories. In: Eggleston H.S., Buendia L., Miwa K., Ngara T. and Tanabe K. (Eds.), National Greenhouse Gas Inventories Programme, IGES, Japão. sem paginação.

Larjavaara, M.; Muller-Landau, H.C. 2010. Rethinking the value of high wood density. Functional Ecology, 24: 701-705.

Lawes, M.J.; Richards, A.; Dathe, J.; Midgley, J.J. 2011. Bark thickness determines fire resistance of selected tree species from fire-prone tropical savanna in north Australia. Plant Ecology, 212: 2057-2069.

Miranda, I.S.; Absy, M.L.; Rebêlo, G.H. 2002. Community structure of woody plants of Roraima savannahs, Brazil. Plant Ecology, 164: 109-123.

Muller-Landau, H.C. 2004. Interspecific and inter-site variation in wood specific gravity of tropical trees. Biotropica, 36: 20-32.

Nogueira, E.; Fearnside, P.; Nelson, B.; Franca, M. 2007. Wood density in forests of Brazil's 'arc of deforestation': Implications for biomass and flux of carbon from land-use change in Amazonia Forest Ecology and Management, 248: 119-135. 
Nogueira, E.; Nelson, B.; Fearnside, P. 2005. Wood density in dense forest in central Amazonia, Brazil. Forest Ecology and Management, 208: 261-286.

Nogueira, E.M.; Fearnside, P.M.; Nelson, B.W. 2008. Normalization of wood density in biomass estimates of Amazon forests. Forest Ecology and Management, 256: 990-996.

Nygård, R.; Elfving, B. 2000. Stem basic density and bark proportion of 45 woody species in young savanna coppice forests in Burkina Faso. Annals of Forest Science, 57: 143-153.

Oliveira, G.M.V.; Mello, J.M.; Trugilho, P.F.; Scolforo, J.R.S.; Altoé, T.F.; Silva-Neto, A.J.; Oliveira, A.D. 2012. Efeito do ambiente sobre a densidade da madeira em diferentes fitofisionomias do estado de Minas Gerais. Cerne, 18: 345-352.

Patiño, S.; Lloyd, J.; Paiva, R.; Baker, T.R.; Quesada, C.A.; Mercado, L.M.; et al. 2009. Branch xylem density variations across the Amazon Basin. Biogeosciences, 6: 545-568.

Reyes, G.; Brown, S.; Chapman, J.; Lugo, A.E. 1992. Wood densities of tropical tree species (SO-88). United States Department of Agricultura, Forest Service, New Orleans. 15 p.

Sarmiento, C.; Patino, S.; Paine, C.E.; Beauchene, J.; Thibaut, A.; Baraloto, C. 2011. Within-individual variation of trunk and branch xylem density in tropical trees. American Journal of Botany, 98: 140-149.

Sternadt, G.H. 2001. Trabalhabilidade de 108 espécies de madeiras da região Amazônica. Instituto Brasileiro do Meio Ambiente e dos Recursos Naturais Renováveis, Laboratório de Produtos Florestais, Brasília, DF, 2001, 106p.

Swenson, N.G.; Enquist, B.J. 2007. Ecological and evolutionary determinants of a key plant functional traits: wood density and its community-wide variation across latitude and elevation. American Journal of Botany, 94: 451-459.

Swenson, N.G.; Enquist, B.J. 2008. The relationship between stem and branch wood specific gravity and the ability of each measure to predict leaf area. American Journal of Botany, 95: 516-519.

Wiemann, M.C.; Williamson, G.B. 1988. Extreme radial changes in wood specific gravity in some tropical pioneers. Wood and Fiber Science, 20: 344-349.

Wiemann, M.C.; Williamson, G.B. 2002. Geographic variation in wood specific gravity: effects of latitude, temperature, and precipitation. Wood and Fiber Science, 34: 96-107.

Williamson, G.B.; Wiemann, M.C. 2010a. Age-dependent radial increases in wood specific gravity of tropical pioneers in Costa Rica. Biotropica, 42: 590-597.

Williamson, G.B.; Wiemann, M.C. 2010b. Measuring wood specific gravity ... Correctly. American Journal of Botany, 97: 519-524.

Williamson, G.B.; Wiemann, M.C. 2011. Age versus size determination of radial variation in wood specific gravity: lessons from eccentrics. Trees, 25: 585-591.

Wittmann, F.; Schöngart, J.; Parolin, P.; Worbes, M.; Piedade, M.T.F.; Junk, W.J. 2006. Wood specific gravity of trees in Amazonian white-water forests in relation to flooding. IAWA Journal, 27: 255-268.

Woodcock, D.W. 2000. Wood specific gravity of trees and forest types in the southern Peruvian Amazon. Acta Amazonica, 30: 589-599.

Recebido em 21/01/2013

Aceito em 22/05/2013 